\title{
PixelFlow: High-Speed Rendering Using Image Composition
}

\author{
Steven Molnar, John Eyles, John Poulton \\ Department of Computer Science \\ University of North Carolina \\ Chapel Hill, NC 27599-3175
}

\begin{abstract}
We describe PixelFlow, an architecture for high-speed image generation that overcomes the transformation- and frame-bufferaccess bottlenecks of conventional hardware rendering architectures. PixelFlow uses the technique of image composition: it distributes the rendering task over an array of identical renderers, each of which computes a full-screen image of a fraction of the primitives. A high-performance image-composition network composites these images in real time to produce an image of the entire scene.
\end{abstract}

Image-composition architectures offer performance that scales linearly with the number of renderers; there is no fundamental limit to the maximum performance achievable using this approach. A single PixelFlow renderer rasterizes up to 1.4 million triangles per second, and an $n$-renderer system can rasterize at up to $n$ times this basic rate.

PixelFlow performs antialiasing by supersampling. It supports deferred shading with separate hardware shaders that operate on composited images containing intermediate pixel data. PixelFlow shaders compute complex shading algorithms and procedural and image-based textures in real-time. The shading rate is independent of scene complexity. A PixelFlow system can be coupled to a parallel supercomputer to serve as an immediatemode graphics server, or it can maintain a display list for retainedmode rendering.

The PixelFlow design has been simulated extensively at high level. Custom chip design is underway. We anticipate a working system by late 1993 .

CR Categories and Subject Descriptors: C.1.2 [Processor Architectures]: Multiprocessors; C.5.4 [Computer System Implementation]: VLSI Systems; I.3.1 [Computer Graphics]: Hardware Architecture; I.3.3 [Computer Graphics]: Picture/Image Generation; I.3.7 [Computer Graphics]: Three-Dimensional Graphics and Realism.

Additional Keywords and Phrases: antialiasing, compositing, deferred shading, rendering, scalable.

Permission to copy without fee all or part of this material is granted provided that the copies are not made or distributed for direct commencial advantage, the ACM copyright notice and the title of the publication and its date appear, and notice is given that copying is by permission of the Association for Computing Machinery. To copy otherwise. or to republish, requires a fee and/or specific permission.

\section{INTRODUCTION}

Graphics applications such as flight and vehicle simulation, computer-aided design, scientific visualization, and virtual reality demand high-quality rendering, high polygon rates, and high frame rates. Existing commercial systems render at peak rates up to 2 million polygons per second (e.g., Silicon Graphics' SkyWriter and Hewlett-Packard's VRX). If antialiasing or realistic shading or texturing is required, however, their performance falls by an order of magnitude.

To support demanding applications, future graphics systems must generate high-resolution images of datasets containing hundreds of thousands or millions of primitives, with realistic rendering techniques such as Phong shading, antialiasing, and texturing, at high frame rates $(\geq 30 \mathrm{~Hz})$ and with low latency.

Attempts to achieve high performance levels encounter two bottlenecks: inadequate floating-point performance for geometry processing and insufficient memory bandwidth to the frame buffer [FOLE90]. For example, to render a scene with 100,000 polygons updated at $30 \mathrm{~Hz}$, geometry processing requires approximately 350 million floating-point operations per second, and rasterization requires approximately 750 million integer operations and 450 million frame-buffer accesses. ${ }^{1}$ Parallel solutions are mandatory.

Most current high-performance architectures use objectparallelism for geometry processing; they distribute primitives over a parallel array of floating-point processors, which perform transformation, clipping, and perspective division [ELLS90; MART90; SGI90].

The same systems use pixel-parallelism for rasterization; framebuffer memory is divided into several interleaved partitions, each with its own rasterization processor. [AKEL88; APGA88; POTM89]. This multiplies the effective frame-buffer bandwidth by the number of partitions, but does not reduce the number of primitives each processor must handle, since most primitives contribute to most partitions [FUCH79]. Because of this limitation, and the bandwidth limitations of commercial VRAMs, this approach does not scale much beyond today's rates of a few million polygons per second.

\footnotetext{
${ }^{1}$ Assumes 50-pixel Gouraud-shaded connected triangles; $3 / 4$ of pixels initially visible; includes screen clear for $1280 \times 1024$ display.
} 


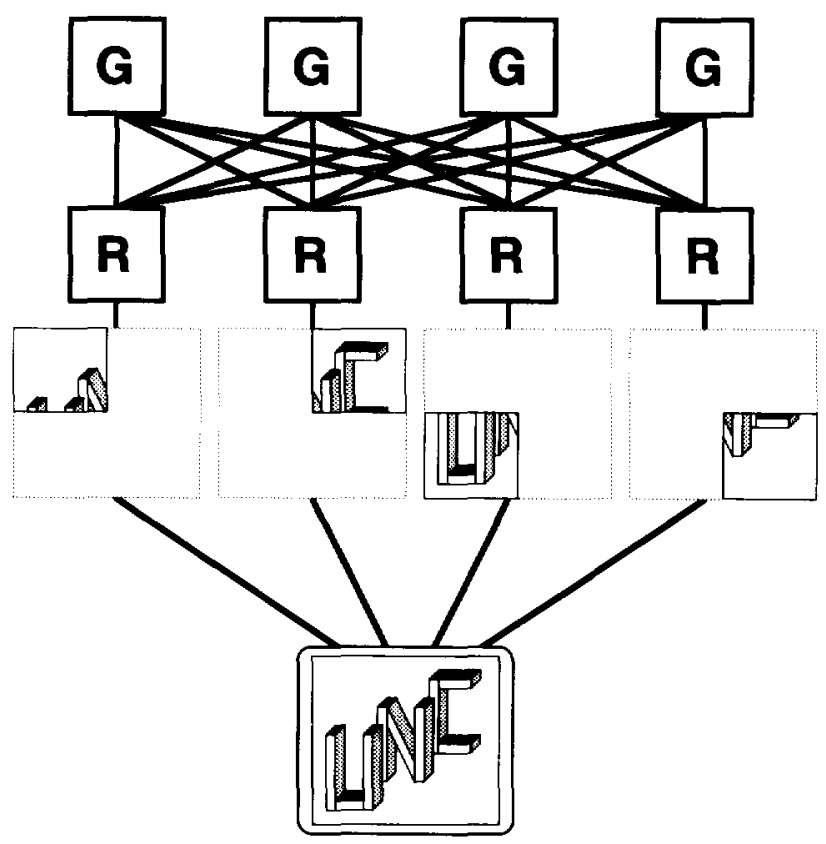

(a) Screen Subdivision

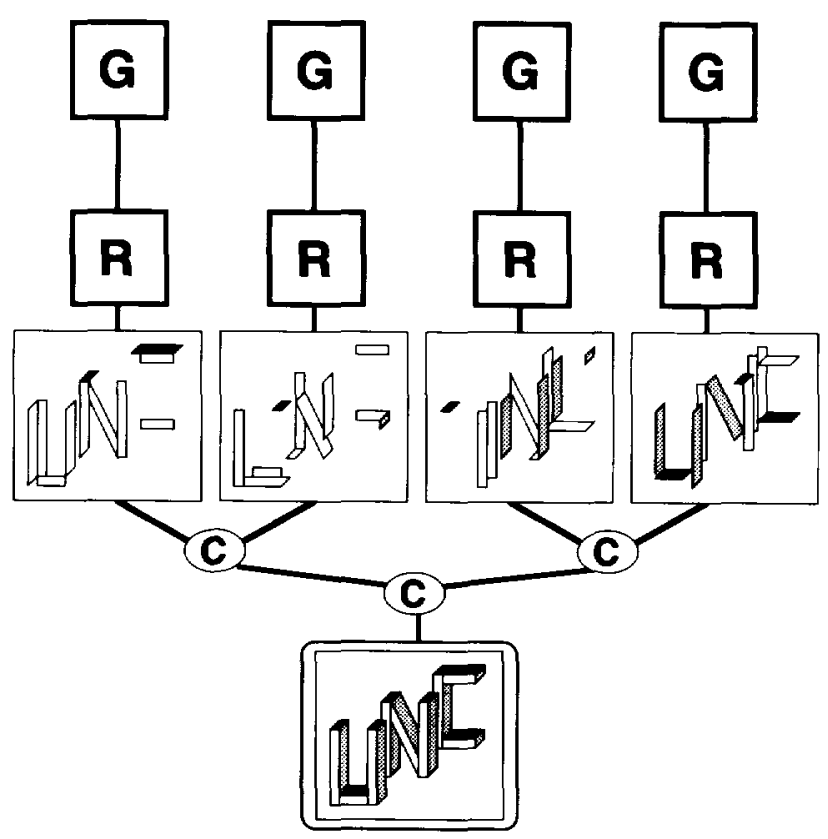

(b) Image Composition

Figure 1: Rasterization methods that combine both pixel-and object-parallel rasterization ( $G=$ geometry processor, $\mathrm{R}=$ rasterizer, and $\mathrm{C}=$ pixel compositor).

To achieve higher rendering performance, object-parallelism must be applied throughout the rendering process - in rasterization, as well as in geometry processing. There appear to be two ways to achieve this: screen subdivision and image composition. The two schemes are shown conceptually in Figure 1.

In the screen-subdivision approach (introduced in [FUCH77] and [PARK80]), the screen is divided into disjoint regions of contiguous pixels, and a rasterization processor is provided for each region (Figure 1a). The processors simultaneously rasterize primitives that fall into different screen regions. These subimages are concatenated to form a full-screen image. Several systems of this type have been described [GARD81; GHAR88] and one built [FUCH89].

This approach is a natural advance from a simple rendering system in which a single geometry processor feeds a single rasterizer. Its main additional requirement is a global routing network to transfer primitives from geometry processors to the appropriate rasterizer. Since the mapping of primitives to rasterizers varies with the viewing transformation, every primitive in the database must be transferred over the network every frame. The network bandwidth is therefore high, and increases linearly with the rendering rate, so the approach does not scale indefinitely.

A second limitation, also due to the global routing network, is the software complexity required to sort primitives by screen region and to route them to rasterizers at the appropriate time. Finally, the architecture is subject to load imbalances when primitives clump into a few screen regions. System performance can decrease significantly in this case.

In the image-composition approach, rasterization processors are associated with a portion of the primitives, rather than with a portion of the screen (Figure lb). Each rasterizer computes a fullscreen image of its portion of the primitives, and these images are composited, based on visibility, to form a final image.

In an image-composition architecture, geometry processors and rasterizers can be paired into self-contained units, so the global routing network for primitives disappears. Instead, an imagecomposition network is required to composite output pixels from the rasterizers to form the final image. This network can be arranged as a binary tree or a pipeline; in either case, all traffic is local, with fixed bandwidth determined by frame rate and screen size. This gives the architecture its property of linear scalability. ${ }^{2}$

The remainder of this paper explores the opportunities and limitations of the image-composition approach. Section 2 discusses image-composition architectures in general. Section 3 introduces PixelFlow, an image-composition architecture that achieves linearly scalable rendering performance and supports high-quality rendering. Sections 4 and 5 describe its major hardware and software components. Section 6 discusses performance issues and presents simulated performance results.

\section{IMAGE COMPOSITION ARCHITECTURES}

Image composition has been used in various forms for many years, particularly in the video industry (e.g., video overlays and chroma-key) and the computer graphics community (e.g., for offline compositing of rendered images).

2 A third method, closely related to image composition, is to provide separate rendering pipelines for multiple image frames. This technique, used in SGI's SkyWriter, multiplies a system's rendering rate and frame rate by the number of rendering pipelines, but does not improve its latency over that of a single pipeline. 
Several graphics architectures based on image composition have been proposed. Processor-per-primitive graphics systems are a simple type of image-composition architecture. [BUNK89] describes one such system, General Electric's 1967 NASA II flight simulator. In the NASA II system, polygons (faces) in the database were assigned to individual processors (face cards). Each face card rendered an image of its respective face, and the results were composited using a static priority scheme.

Later researchers proposed using $z$-values to determine the image priority at individual pixels [DEME80; FUSS82]. [WEIN81] proposed an antialiasing scheme for a processor-per-primitive system. [DEER 88] and [SCHN88] proposed deferred shading as a way to support high-quality shading in a processor-per-primitive system. [ELL191] describes a processor-per-primitive system specialized for CSG rendering, which was built at Duke University and UNC.

A few image-composition architectures with multi-primitive renderers have been proposed: [SHAW88] described a simplified version of Duff's compositing algorithm [DUFF85] cast in VLSI to create a multi-renderer system that performs antialiasing. [MOLN88] proposed a simple z-buffer image-composition scheme to achieve linearly scalable rendering performance; this idea was expanded into a dissertation on image-composition architectures [MOLN91b], which also describes an early version of the PixelFlow architecture.

\subsection{Advantages and Disadvantages}

Image-composition offers two potential advantages over other architectural approaches: linear scalability and a simple programming model. An arbitrary number of renderers can be added to the system. since the image-composition network has only local traffic with fixed bandwidth determined by screen size and frame rate. Also, since renderers compute their sub-images independently, they can operate with little synchronization. This makes the parallel nature of the system largely transparent to the programmer.

Image-composition architectures have several disadvantages, however. First, the image-composition network must support very high bandwidth communication between renderers. Even though the bandwidth is fixed, the network must transfer every pixel during every frame, and each pixel must include visibility information and color (and possibly even more data, if deferred shading is used). Second, pixels must be reduced to a common format for compositing, so the visibility algorithm is more restrictive than in some other approaches. Finally, up to a frame of pixel storage is required per renderer, if an entire frame is buffered before being composited; fortunately, this storage requirement can be reduced, as we will see in Section 3 .

\subsection{Antialiasing}

Aliasing artifacts, once ubiquitous in interactive systems, are tolerated less and less each year. Future real-time systems must provide ways to reduce or eliminate these artifacts. There appear to be two ways to combat aliasing in image-composition systems: supersampling, and A-buffer algorithms [MOLN9Ib]. In the supersampling approach, the image is generated and composited multiple times, once for each sample in a filter kernel. The compositors perform a simple z comparison for each subpixel; then subpixels are blended together after composition to form a final image. In the A-buffer approach, each pixel is represented by a variable-length packet describing all surfaces potentially visible at the pixel. Compositors merge packets together based on visibility and coverage information. The output of the network describes all of the surfaces contributing to each pixel, and this information is used to compute the pixel's final color.

In comparing the two approaches, the critical factors are image quality, image-composition bandwidth, and hardware complexity. Supersampling produces good results, provided that sufficient samples are taken per pixel. Unfortunately, the number of samples directly affects the bandwidth required of the imagecomposition network; however, we have produced reasonablequality images with as few as 5 samples per pixel by choosing sample locations and weights carefully [MOLN91a].

Only the simplest A-buffer methods are feasible in current realtime systems. These methods generally sample color and $z$ values at pixel centers only, while calculating pixel coverage at higher resolution. This can lead to artifacts. To avoid these artifacts, additional information must be added to the surface descriptors; the result is that the two approaches require comparable bandwidth. In terms of hardware complexity, A-buffer renderers and compositors are fairly complex, while the $z$-depth compositors for supersampling are very simple. The A-buffer approach supports true transparency, however, which is problematic in the supersampling approach

\subsection{Deferred Shading}

Image-composition architectures can take special advantage of deferred shading, a general method for reducing the calculations required for complex shading models by factoring them out of the rasterization step [DEER88; ELLS91]. Many shading calculations depend only on geometric and intrinsic attributes, such as surfacenormal vectors and surface color. If these attributes are calculated and stored during rasterization, shading can be deferred until the entire scene has been rasterized, and applied only to the surfaces that are visible.

To defer shading in an image-composition system, rasterizers compute generic pixel attributes and composite these, rather than pixel colors. A separate hardware unit performs shading after pixels have been composited. In this manner, shading is performed just once per pixel, no matter how many surfaces contribute to it and how many rasterizers are in the system. Deferred shading does increase image-composition bandwidth, however, since the number of bits per pixel is generally higher.

Deferred shading also separates performance concerns between the rendering and shading portions of the machine. Renderers can be built to rasterize as fast as possible, and the number of renderers can be chosen to achieve a given polygon rate. Shading performance is independent of the rasterizing performance of the system, but most be high enough to support the desired shading model.

\section{PIXELFLOW ARCHITECTURAL OVERVIEW}

PixelFlow is an experimental graphics system designed to demonstrate the advantages of image-composition architectures and to provide a research platform for real-time $3 \mathrm{D}$ graphics algorithms and applications. In this section we describe its major architectural features and the rationale under which they were chosen. Section 4 provides details of system components. 
Supersampling antialiasing. PixelFlow uses the supersampling approach because it is general, the compositor hardware is simple, and therefore fast, and it can be tuned to trade speed for image quality. This leads to renderers based on $z$-buffer rasterizers and a $z$-based image-composition network. Unfortunately, this requires screen-door or multi-pass algorithms to support transparency.

Pipelined image-composition network. Generating Gouraudshaded, supersampled, high-resolution images at $30 \mathrm{~Hz}$ frame rates requires composition-network bandwidth of at least $1280 \times 1024$ pixels $\cdot 5$ samples/pixel $\cdot 48$ bits/sample $\cdot 30$ frames/second $=9.4 \mathrm{Gbits} /$ second. Deferred shading algorithms require 2 to 3 times this amount.

Packaging considerations favor a pipeline image-composition network. The image-composition network can be distributed across the system by including a compositor on each board and daisy-chaining connections between boards.

Logic-enhanced memory rasterizer. The renderer should a single-board design, must provide a way to scan out pixels at the bandwidth required, and should implement the compositor function at relatively low cost in board area, power, and dollars. These considerations mainly affect the design of the rasterizer.

The logic-enhanced memory approach used in Pixel-Planes 5 allows a powerful rasterizer and high-bandwidth compositor to be built in a single, compact package. In PixelFlow, we use a similar logic-enhanced memory approach. A rasterizer built with new PixelFlow enhanced-memory chips (EMCs) can render in excess of one million triangles per second and provide imagecomposition bandwidth exceeding 30 Gbits/second using 64 custom memory chips and one custom controller on about 50 square inches of board area.

Region-based rendering scheme. The compactness of this approach is obtained at the cost of introducing screen subdivision at the level of the individual renderers. As in Pixel-Planes 5, each rasterizer contains only $128 \times 128$ pixel processors, and must generate a full-screen image in multiple steps. The advantage is that an entire screen's worth of pixel memory is not required. Unfortunately, this implementation incurs the load-balancing problems of screen subdivision, but these difficulties are greatly reduced by providing several region's worth of buffering within the PixelFlow EMCs

The required image-composition bandwidth is achieved in two ways. First, the network operates bit-serially, but in parallel on 256 pixels, each with its own single-wire channel. Bit-serial $z$ comparison simplifies the compositors and thereby allows them to operate at high speed. Second, the network consists entirely of point-to-point communication between identical custom chips on neighboring boards, so low voltage swings and source termination can be used to save power and provide the necessary speed (132 $\mathrm{MHz}$ ) [KNIG88].

Separate shaders for deferred shading. Deferred shading algorithms, such as Phong shading and procedural and imagebased textures, are implemented on separate hardware shaders that reside just ahead of the frame buffer. Regions of pixels, containing attributes such as intrinsic color, surface normals, and texture coordinates are rasterized on the renderers, composited on the image-composition network, and loaded into the shaders. Shaders operate on entire regions in parallel, to convert raw pixel attributes into final RGB values, blend multiple samples together for antialiasing, and forward final color values to the frame buffer.
Regions are assigned to shaders in round-robin fashion. The number of shaders required depends on the shading algorithm only, not on the number of primitives, since deferred shading is employed.

The SIMD rasterizer used in the renderer is an ideal processor for deferred shading, since shading calculations can be performed for all pixels simultaneously. Therefore, the shaders can simply be designated renderers, with a slight enhancement of the compositor hardware on the EMC to allow bidirectional data transfers between the image-composition network and EMC memory. Shaders can be augmented with additional hardware to allow them to compute image-based textures in addition to procedural textures.

\section{PIXELFLOW HARDWARE}

A PixelFlow system is composed of one or more card cages, each containing up to 20 circuit boards. The backplane contains wiring for the image-composition network and clock and power distribution. The system is modular and can be configured with any number of card cages. Each board has a high-speed link connecting it to a host computer. Figure 2 shows a block diagram of a PixelFlow system.

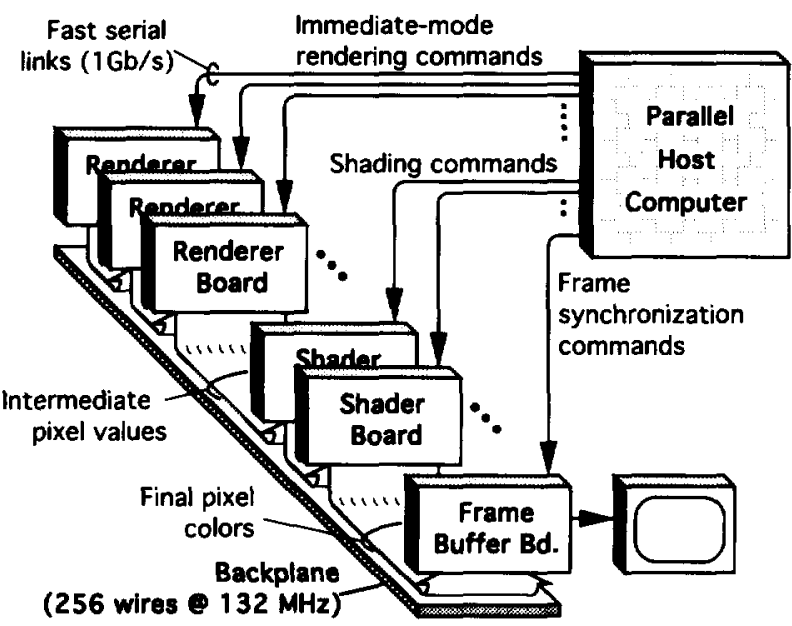

Flgure 2: Block diagram of a PixelFlow system.

Renderers operate by sequentially processing $128 \times 128$-pixel regions of the screen. They scan out the region's rasterized pixels over the image-composition network in synchrony with the other renderers. Shaders load pixels from the image-composition network, perform texturing and shading, blend subpixel samples, and forward pixel values to the frame buffer.

The system is designed to be used in one of two basic modes:

1) Immediate Mode. PixelFlow is hosted by a parallel computer, with each link connected to a separate compute node in the host. The host (e.g., an Intel Touchstone) runs an application and generates immediate-mode primitives, which are transmitted to renderers over the links.

2) Retained Mode. PixelFlow is hosted by a workstation; the high-speed links are bussed together and connected to the host via a single interface. The host distributes a display 


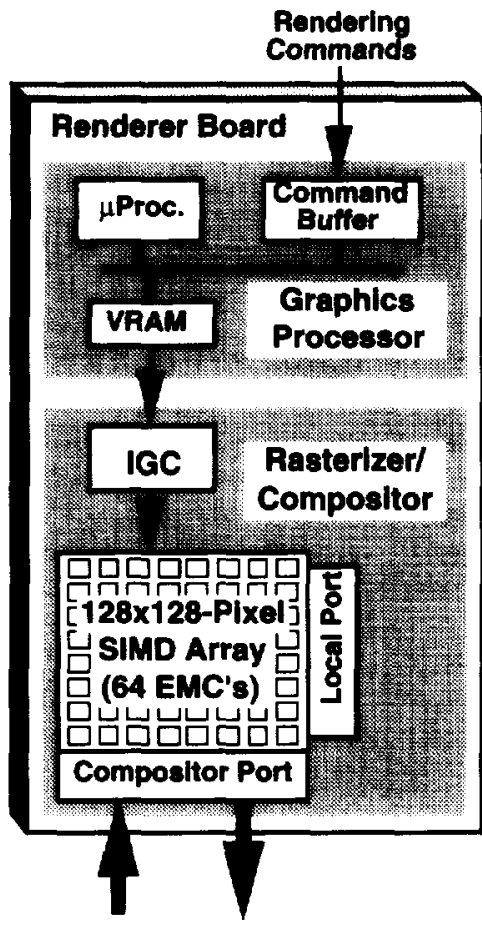

(a)

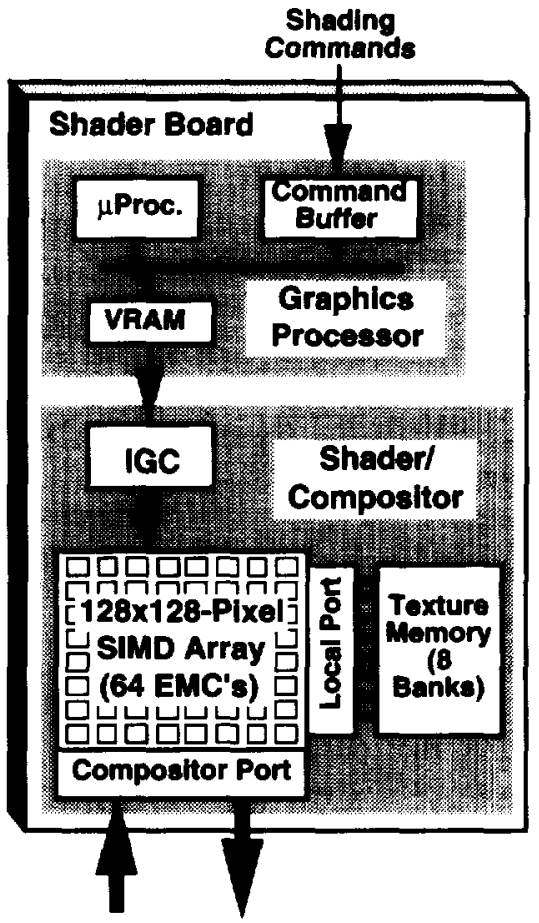

(b)

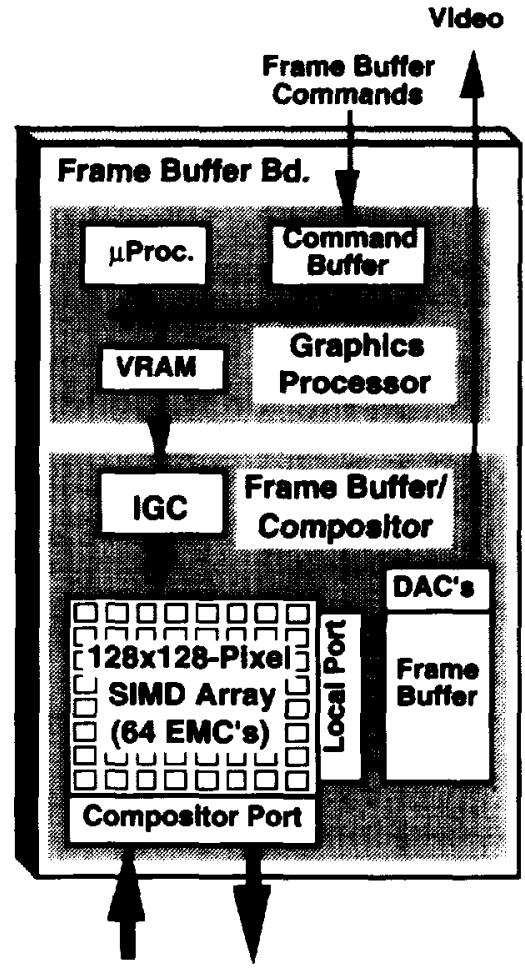

(c)

Figure 3: Block diagrams of (a) renderer, (b) shader, and (c) frame-buffer boards.

list over the renderers and loads a shading model into the shaders. On each frame, the host broadcasts editing commands and viewing parameters to the renderers, each of which then computes an image of its fraction of the dataset.

Each of the primary board types (renderers, shaders, and frame buffers) includes a core consisting of a geometry processor and a rasterizer built from the new EMC chips. These core elements have different function on the three board types, and shaders and frame buffers contain additional components (Figure 3).

\subsection{Image-Composition Network}

The image-composition network is a wide (256-bit), high-speed (132 $\mathrm{MHz}$ ) special-purpose communication network for rapidly moving pixel data between boards. It is distributed across the EMCs on each board, with each EMC implementing a 4-bit-wide slice ( 4 input pins and 4 output pins). Compositors on the EMCs synchronously transmit data unidirectionally to the compositors on the downstream board.

Compositor modes. The basic unit of operation is the transfer of one 128x128-pixel region of pixel data; the amount of data transferred per pixel is preset according to the specific algorithm. The compositors operate in one of four modes, as shown in Figure 4.

\subsection{Renderer}

The renderer block diagram is shown in Figure 3a. Its major components are:
Geometry processor. The geometry processor is a fast floating point processor that retains a portion of a distributed display list (retained mode) or receives a fraction of the primitives from the host on each frame (immediate mode). It transforms its portion of the primitives into screen coordinates, sorts them by screen region, and passes them to the rasterizer. It contains $8 \mathrm{MBytes}$ of VRAM memory, serving both as main memory and as a large FIFO queue for buffering commands to the rasterizer. A DMA engine controls the flow of commands from the VRAMs' serial port to the rasterizer, maintaining separate queues of commands for rasterization and for transfers over the image-composition network.

Rasterizer. The rasterizer is a $128 \times 128$ SIMD processor array implemented with 64 PixelFlow EMCs driven by instructions and data broadcast from an Image Generation Controller (IGC) ASIC.

The PixelFlow EMC (Figure 5) is similar to our previous designs [EYLE88; POUL85]. A linear expression evaluator computes values of the bilinear expression $A x+B y+C$ at every pixel processor in parallel $(x, y$ is the pixel processor's screen location and $A, B$, and $C$ are user-specified). Each pixel has a small local ALU that performs arithmetic and logical operations on local memory and on the local value of the bilinear expression. Operation of the pixel processors is SIMD (single-instructionmultiple-data), and all processors operate on data items at the same address. Each pixel processor includes an enable register which qualifies writes to memory, so that a subset of the processors can be disabled for certain operations (e.g. painting a scan-converted polygon). 


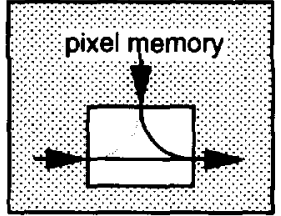

Composite local pixels with upstream pixels.

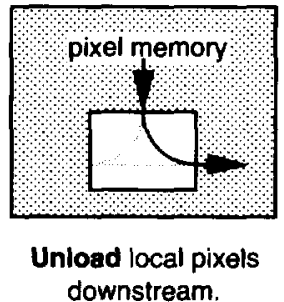

Figure 4: Compositor operating modes.

Several features distinguish the PixelFlow EMC from our previous designs:

- Higher clock speed. The new EMC, fabricated on a $0.8 \mu$ CMOS process, operates at $66 \mathrm{MHz}$ for image-generation operations; its image-composition port transmits data at $132 \mathrm{MHz}$

- 8-bit processors. The pixel processors, linear expression evaluator, and memory bus for each pixel are eight bits wide, rather than bit-serial. This increases the performance for many operations by nearly a factor of eight.

- Fast multiply hardware. Pixel processors include hardware support for multiplies, allowing 16-bit multiplies to be performed in less than a microsecond--a total of 19 billion multiplies per second for a $128 \times 128$-pixel array. This feature accelerates multiply-intensive shading calculations.

- 2048+ bits per pixel. The memory design uses a 1transistor dynamic memory cell [SPEC91], rather than the 6-transistor static memory cell used previously. Memory per pixel can be increased to 2048 bits, plus two 256-bit communication buffers.

- Compositor and local-access ports. The PixelFlow EMC contains two communication ports, one for the image-composition network, and one for communicating with texture memory or a frame buffer. Each port contains a 256-bit buffer of pixel memory that can be read or written by the pixel ALU or decoupled from it during port operation. The local-port connects to external texture memory (on a shader board) or a VRAM frame store (on a frame-buffer board) through a custom datapath ASIC.

The IGC is a single custom ASIC which controls the rasterizer array. It converts floating-point $A, B$, and $C$ coefficients into byteserial, fixed-point form; it sequences EMC operations by broadcasting data, control, and address information to the EMC array; and it controls the compositor ports on the EMCs.

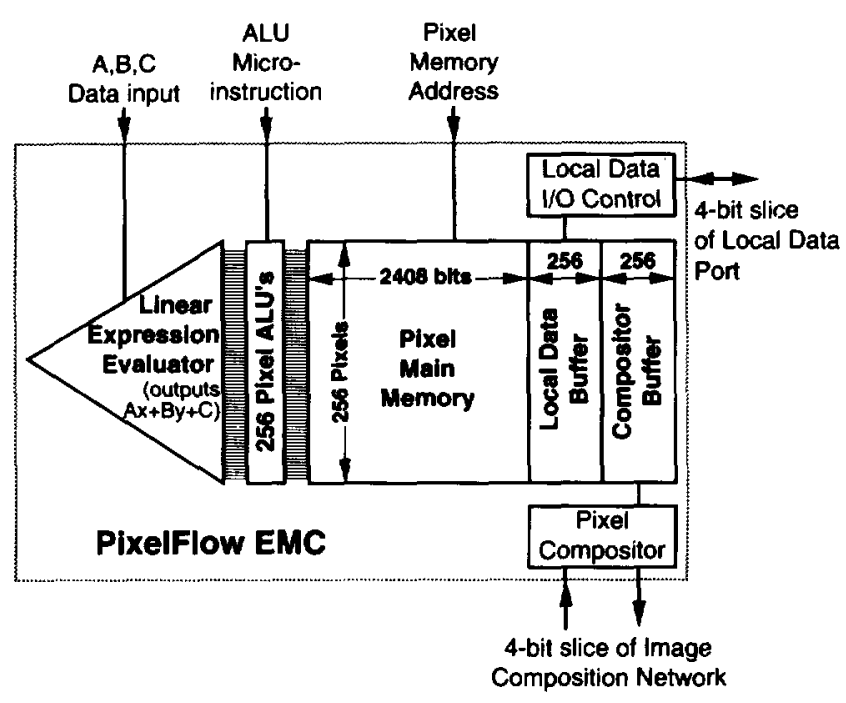

Figure 5: Block diagram of PixelFlow Enhanced Memory Chip.

The IGC contains a subpixel offset register that allows the multiple samples of the supersampling filter kernel to be computed from the same set of rasterization commands, by repeatedly reading these commands from VRAM memory. This improves system performance when supersampling, since additional samples are rasterized without increasing the load on the geometry processor. As a result, a single i860XP geometry processor can keep up with the rasterizer when supersampling with 6 or more samples per pixel.

\subsection{Shader}

The shader (Figure $3 \mathrm{~b}$ ), like the renderer, contains a geometry processor, rasterizer, and compositor. The shader's geometry processor is merely a control processor which passes shading commands to the rasterizer. The rasterizer is used as a SIMD shading processor, computing lighting and shading models for all pixels in a region in parallel. The compositors are used to load composited regions and unload fully-shaded pixels.

The local communication port of the EMCs is connected to external memory that contains image-based textures (such as Mipmaps). Multiple banks of texture memory, each holding an identical copy of the texture data, are required to match the performance of the shader to that of the image-composition network. The shader supports general table-lookup operations, so it can perform related functions such as bump mapping, environment mapping, and image warping. The shader can be loaded with an image, from which it computes a Mip-map that can then be loaded into texture memory. These algorithms will be described in a future publication.

\subsection{Frame Buffer}

The frame buffer (Figure 3c) closely resembles the shader, but in place of texture storage, it contains a separate double-buffered VRAM frame buffer. The frame buffer board is itself a complete, fairly powerful, self-contained graphics system, since it also contains the core elements (geometry processor and rasterizer). 


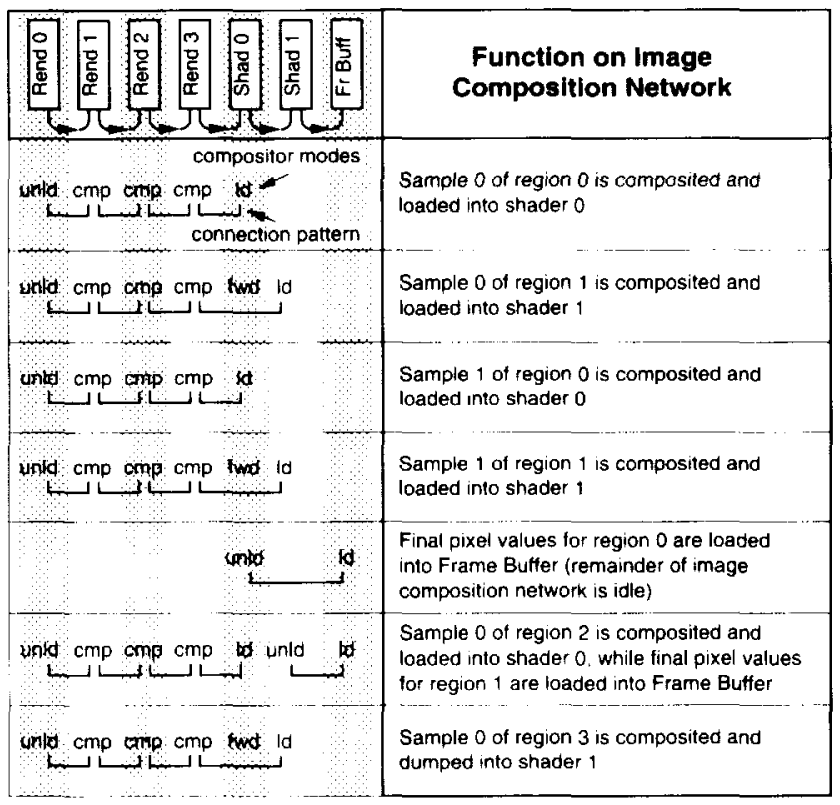

Figure 6: Composition network operations for a 4renderer, 2-shader system computing an image with 2sample-per-pixel antialiasing.

\section{SOFTWARE}

PixelFlow software consists of two parts: rendering software, which transforms primitives and produces rasterization/shading commands for the rasterizer, and control software, which sequences region-by-region operations. Both sets of software run on the geometry processor of each system board.

Geometric transformations are performed using standard algorithms, and rasterization and shading are performed using the algorithms developed for Pixel-Planes 4 and 5 [FUCH85: FUCH89].

The basic control algorithm to compute an image contains four steps:

1) The geometry processor on each renderer transforms its portion of the primitives and sorts them by $128 \times 128$-pixel regions on the screen.

2) The rasterizer computes pixel values for all primitives in a region and for one sample of the antialiasing kernel.

3) When all renderers have finished step (2), the region is composited over the image-composition network and deposited into one of the shaders.

4) Steps (2) and (3) are repeated for each sample and for each region on the screen. These steps can be pipelined (i.e. one region/sample is rasterized while the previous one is composited).

Transfers over the image-composition network are the only operations that require tight synchronization between boards. A hardware token chain determines when all of the boards are ready to begin each transfer. Figure 6 shows the sequence of composition network operations in a 4-renderer, 2 -shader system computing an image with 2-sample-per-pixel antialiasing.
The large amount of pixel memory on the EMCs allows several regions of pixels to be buffered before they are composited. This is important for load balancing, since different numbers of primitives may fall into a given region on different renderers (see Section 6). To take full advantage of this buffering, regions are processed in a scattered pattern, since neighboring regions tend to have similar over- or underloads; successive samples of a given region are never handled sequentially.

\section{PERFORMANCE}

The performance of a PixelFlow system is governed by four basic parameters:

- Image-Composition Network bandwidth. Gross bandwidth $=256$ bits $\cdot 132 \mathrm{MHz}=33.8 \mathrm{GBits} / \mathrm{sec}$. Net bandwidth (assuming 10\% synchronization ovèrhead) $=30$ GBits/sec.

- Geometry processor performance. A single i860XP microprocessor can process approximately 150,000 triangles per second, independent of the number of samples per pixel.

- Rasterizer performance. A 64-EMC rasterizer can process approximately 1.4 million Gouraud-shaded triangles per second and 0.8 million Phong-shaded, textured triangles per second, but this rate must be divided by the number of samples per pixel.

- Shader Performance. A single shader can Phong-shade and compute procedural textures for approximately 10,000 $128 \times 128$-pixel regions per second. It can compute Mipmap textures for approximately 3,700 regions per second

The following expression can be used to estimate system performance given the performance above:

$$
T_{\text {frame }}=\sum_{i=1}^{N_{\text {regions }}} \max \left(T_{\text {rend }}, T_{\text {comp }}, T_{\text {shade }} / N_{\text {shaders }}\right)
$$

where

$$
\begin{aligned}
& T_{\text {rend }}=\max _{\left.\operatorname{Region}_{i}\right)}\left(T_{\text {geom }_{i}}, T_{\text {rast }_{i}}\right) \text { (the rendering time for } \\
& T_{(r) m p}=\text { compositing time for a region }\left(128^{2} \text { pixels } \cdot\right. \text { bits } \\
& \text { per pixel } 30 \mathrm{GBits} / \mathrm{sec} \text { ) } \\
& T_{\text {shade }}=\text { time to shade a region (approx. } 270 \mu \mathrm{sec} \text { for } \\
& \text { Phong shading and texturing) }
\end{aligned}
$$

If antialiasing is done, the summation is over all regions and over all antialiasing samples.

This equation says that the frame time is given by the sum of the times required to compute each region, and that each region time is given by the maximum of the geometry processing/rasterization time, image composition time, and shading time for the region. Both $T_{c o m p}$ and $T_{\text {shade }}$ are constants, for a given rendering algorithm. Trend, varies depending on the number of renderers, the number of primitives. and their distribution over the renderers and over the screen. 
$T_{\text {comp }}$ provides an upper bound on frame rate, as shown in Figure 7 for several rendering algorithms and system configurations.

\begin{tabular}{lccc} 
Bits per pixel & Samples & Screen & Frame/sec \\
\hline 64 (Gouraud) & 16 & $1280 \times 1024$ & 22 \\
96 (Phong) & 1 & $1280 \times 1024$ & $>100$ \\
96 (Phong) & 5 & $1280 \times 1024$ & 48 \\
192 (Phong, textured) & 5 & $1280 \times 1024$ & 24 \\
192 (Phong, textured) & 16 & $640 \times 512$ & 29
\end{tabular}

Figure 7: Peak performance dictated by compositionnetwork bandwidth under varying conditions.

Actual system performance can be lower than predicted by this equation because of several factors: First, primitives may cross region boundaries and require processing in multiple regions. This increases the rasterization load by approximately $20 \%$ for 100 -pixel polygons. Second, primitives may clump in different screen regions on different renderers. This increases the rasterization time on certain renderers relative to others. The extra buffering on the EMCs reduces this effect, but does not eliminate it entirely. For severely clumped images, system performance can be reduced from $20-60 \%$. Finally, rasterization and compositing are pipelined, so that one region is rasterized while the previous one is composited. This requires a few idle region times at the start and end of a frame (approximately $5-10 \%$ overhead).

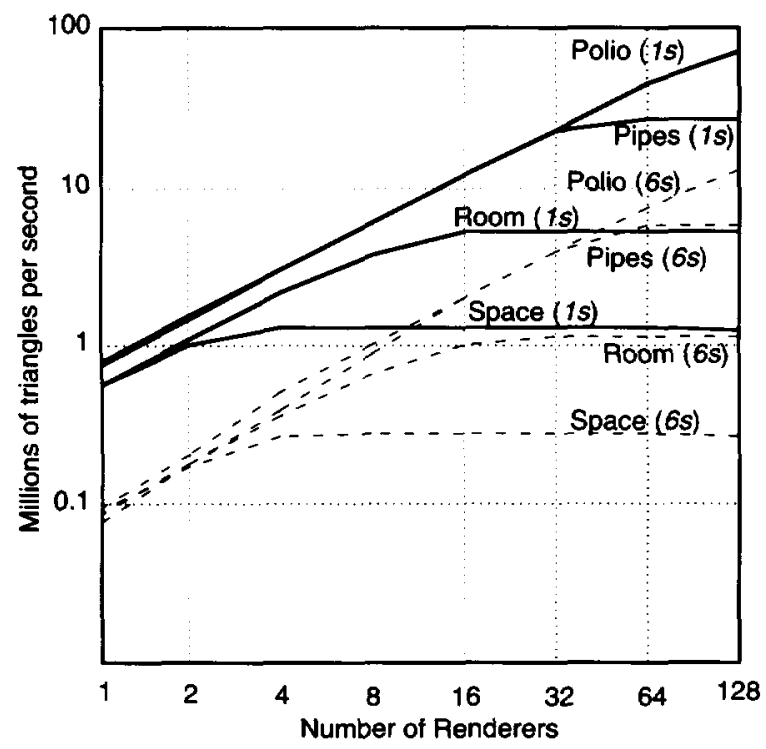

Figure 8: Simulated performance for various system configurations.

Because these factors are scene dependent, they cannot be modelled analytically. We have written a timing simulator for PixelFlow that models these effects and have used it to compute performance for a variety of images and rendering algorithms. Figure 8 shows simulated performance for the four sample databases shown in Figure 9. Simulations were run with 1 to 128 renderers and 4 shaders. Two curves are shown for each dataset: one for a supersampled image with 6 samples per pixel $(6 s)$, and one for a "fully-aliased" image with one sample per pixel $(1 s)$. For the $6 s$ case, we assumed the geometry processor is a $66-\mathrm{MHz}$ i860XP; for the $1 s$ case, we assumed that a sufficiently powerful geometry processor is available so that renderer performance is rasterizer-limited.

These simulated results show the behavior predicted by the equation above. System performance scales linearly with the number of renderers until a knee is reached, where compositing time dominates rasterization time (shading time is not a limiting factor for any of these datasets). The space station dataset, in particular, is very small ( 3,784 primitives), so this knee is reached at only 4 renderers. Only the polio dataset is large enough to show linear scalability to 128 renderers.

\section{CONCLUSIONS}

We have introduced PixelFlow, a new architecture for high-speed image generation, and one of the first to use real-time image composition with multi-primitive renderers. Its combination of million-triangle-per-second renderers and high-performance compositing network give it linearly scalable performance to tens of millions of polygons per second- far above the performance of current systems.

All of the components of PixelFlow are programmable: its geometry processors are conventional microprocessors; its rasterizers are programmable SIMD processors; its imagecomposition network is a general pixel datapath. In addition to standard rendering algorithms, such as Gouraud- and Phongshading of polygonal primitives, PixelFlow can render primitives such as spheres, quadrics, and volume data with high-quality shading methods, such as local light sources, procedural and image-based texturing, and environment mapping.

A PixelFlow system can be configured in a variety of ways. Hosted by a single workstation, it can render PHIGS-type retained-mode datasets. Coupled to a parallel supercomputer, it can serve as a visualization subsystem for immediate-mode rendering. Using PixelFlow silicon, a million-triangle-per-second rasterizer could be built on a small circuit board.

At the time this paper was written, logic design for the custom chips was nearly complete. We anticipate completing circuit design for the system by mid-1993 and completing a prototype system by late 1993 .

\section{ACKNOWLEDGEMENTS}

We acknowledge the following people for their suggestions and contributions to this work: Henry Fuchs, Turner Whitted, Anselmo Lastra, Jon Leech, Brice Tebbs, Trey Greer, Lee Westover, and the entire Pixel-Planes team.

This research is supported in part by the Defense Advanced Research Projects Agency, DARPA ISTO Order No. 7510, and the National Science Foundation, Grant No. MIP-9000894. 


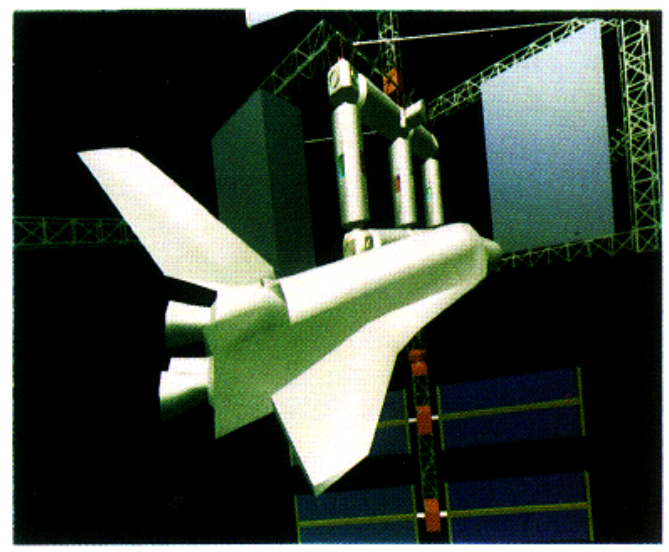

(a) Space station and space shuttle, Phongshaded, 6,549 triangles (Don Eyles, Charles Stark Draper Labs).

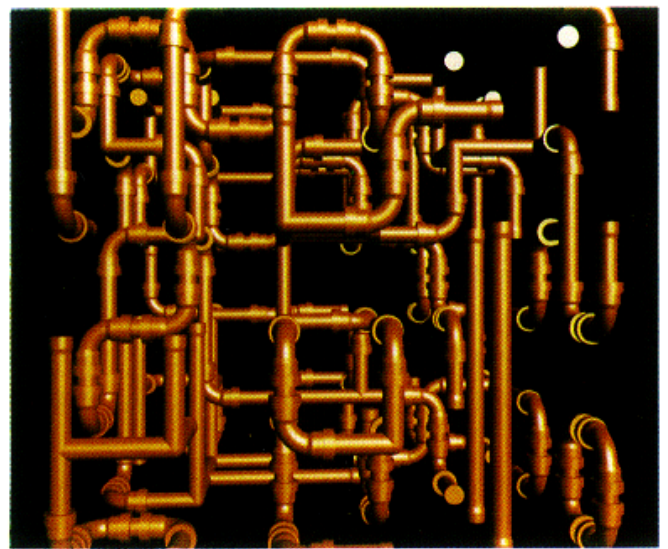

(c) Procedurally generated pipes model, Phong-shaded, 137,747 triangles (Lee Westover, Sun Microsystems).

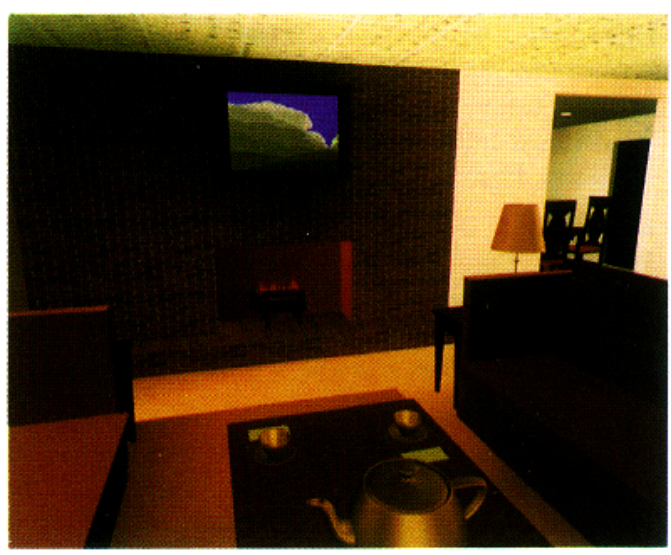

(b) Radiosity-shaded room interior with procedural textures, 53,514 triangles (F.P. Brooks, A. Varshney, UNC).

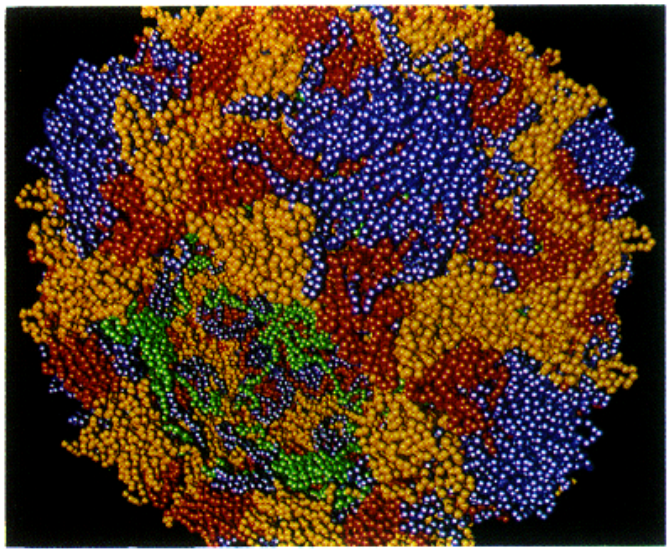

(d) Poliovirus molecule, Phong-shaded, 389,819 triangles (J. Hogle, M. Chow, D. Filman, Scripps Institute).

Figure 9: Four sample datasets used for timing simulation.

\section{REFERENCES}

AKEL88 Akeley, K. and T. Jermoluk, "High-Performance Polygon Rendering," SIGGRAPH' '88, Vol. 22, No. 4, pp. 239-246.

APGA88 Apgar, B.. B. Bersack, and A. Mammen, "A Display System for the Stellar Graphics Supercomputer Model GS 1000," SIGGRAPH'88, Vol. 22, No. 4, pp. 255-262.

BUNK89 Bunker, M. and R. Economy. "Evolution of GE CIG Systems," SCSD Document, General Electric Company, Daytona Beach, FL 32015, 1989.

DEER88 Deering, M., S. Winner, B. Schediwy, C. Duffy, and N. Hunt. "The Triangle Processor and Normal Vector Shader: A VLSI System for High Performance Graphics," SIGGRAPH '88, Vol. 22, No. 4, pp. 2130.
DEME80 Demetrescu, S., A VLSI-Based Real-Time Hidden Surface Elimination Display System, Master's Thesis, Dept. of Computer Science, California Institute of Technology, 1980.

DUFF85 Duff, T., "Compositing 3D Rendered Images," SIGGRAPH '85. Vol. 19. No. 3, July 1985, pp. 41-44.

ELL191 Ellis, J., G. Kedem, T. Lyerly, D. Thielman, R. Marisa, J. Menon, H. Voelcker, "The Raycasting Engine and Ray Representation: A Technical Summary," Proc. of the Intl. Jornal of Computational Geometry and Applications, 1991.

ELLS90 Ellsworth, D.E., H. Good, and B. Tebbs, "Distributing Display Lists on a Multicomputer," Computer Graphics (Proceedings of the 1990 Symposium on Interactive 3D Graphics), Vol. 24, No. 2, March 1990. p.147-154. 
ELLS91 Ellsworth, D.E. "Parallel Architectures and Algorithms for Real-Time Synthesis of High-Quality Images Using Deferred Shading," Workshop on Algorithms and Parallel VLSI Architectures, Pont-àMousson, France, June 12, 1990.

EYLE88 Eyles, J., J. Austin, H. Fuchs, T. Greer, and J. Poulton, "Pixel-Planes 4: A Summary," $A d v$. in Computer Graphics Hardware II (1987 Eurographics Workshop on Graphics Hardware), Eurographics Seminars, 1988, pp. 183-208.

FOLE90 Foley, J.D., A. van Dam, S.K. Feiner, and J.F. Hughes, Computer Graphics: Principles and Practice, Addison-Wesley, Reading, MA, 1990. (especially Chapter 18, "Advanced Raster Graphics Architecture")

FUCH77 Fuchs, H., "Distributing a Visible Surface Algorithm over Multiple Processors," Proceedings of the ACM Annual Conference, pp. 449-451.

FUCH79 Fuchs, H. and B. Johnson, prepublication draft of "An Expandable Multiprocessor Architecture for Video Graphics," Proceedings of the 6th ACM-IEEE Symposium on Computer Architecture, April, 1979, pp. 58-67.

FUCH85 Fuchs, H., J. Goldfeather, J. Hultquist, S. Spach, J. Austin, F. Brooks, J. Eyles, and J. Poulton, "Fast Spheres, Shadows, Textures, Transparencies, and Image Enhancements in Pixel-planes," SIGGRAPH '85, Vol. 19, No. 3, pp. 111-120.

FUCH89 Fuchs, H., J. Poulton, J. Eyles, T. Greer, J. Goldfeather, D. Ellsworth, S. Molnar, G. Turk, B. Tebbs, and L. Israel, "Pixel-Planes 5: A Heterogeneous Multiprocessor Graphics System Using Processor-Enhanced Memories," SIGGRAPH '89, Vol. 23, No. 3, pp. 79-88.

FUSS82 Fussel, D. and B. D. Rathi, "A VLSI-Oriented Architecture for Real-Time Raster Display of Shaded Polygons." Graphics Interface '82, 1982, pp. 373380

GARD81 Gardner, G.Y., E.P. Berlin, Jr., and B.M. Gelman, "A Real-Time Computer Image Generation System using Textured Curved Surfaces," Proceedings of IMAGE $I I, 1981$, pp. 59-76.

GHAR88 Gharachorloo, N., S. Gupta, E. Hokenek, P. Balasubramanian, B. Bogholtz, C. Mathieu, and C. Zoulas, "Subnanosecond Pixel Rendering with Million Transistor Chips," SIGGRAPH 88, Vol. 22, No. 4, pp. 41-49.

KNIG88 Knight, T. and A. Krimm, "A Self-Terminating LowVoltage Swing CMOS Output Driver," IEEE Journal of Solid-State Circuits, Vol. 23, No. 2, April 1988, pp. 457-464.

MART90 Martin, P., and H. Baeverstad, "TurboVRX: A HighPerformance Graphics Workstation Architecture," Proc. of AUSGRAPH 90, September 1990, pp. 107117.

MOLN88 Molnar, S.E., "Combining Z-buffer Engines for Higher-Speed Rendering," Advances in Computer Graphics Hardware III, Eurographics Seminars, 1988, pp. 171-182.
MOLN9la Molnar, S.E., "Efficient Supersampling Antialiasing for High-Performance Architectures," Technical Report TR-91-023, Dept. of Computer Science, UNC-Chapel Hill, 1991

MOLN91b Molnar, S.E., "Image Composition Architectures for Real-Time Image Generation," Ph.D. dissertation, also available as UNC-Computer Science Technical Report TR91-046, 1991

PARK80 Park, F., "Simulation and Expected Performance Analysis of Multiple Processor Z-Buffer Systems," SIGGRAPH' 80 , Vol. 14, No. 3, pp. 48-56.

POTM89 Potmesil, M., and E. Hoffert, "The Pixel Machine: A Parallel Image Computer," SIGGRAPH '89, Vol. 23, No. 3, pp. 69-78.

POUL85 Poulton, J., H. Fuchs, and A. Paeth, "Pixel-planes graphic engine," Section 9.5 in Principles of CMOS VLSI Design: A System Perspective, by Neil Weste and Kamran Eshrahian, Addison-Wesley, New York, 1985, pp. 448-480.

SGI90 Silicon Graphics Computer Systems, Vision Graphics System Architecture, Mountain View, CA 94039. 7311 , February 1990.

SHAW88 Shaw, C.D., M. Green, and J. Schaeffer, "A VLSI Architecture for Image Composition," Advances in Computer Graphics Hardware III, Eurographics Seminars, 1988, pp. 183-199.

SPEC91 Speck, D., "The Mosaic Fast 512K Scalable CMOS DRAM," Proceedings of the 1991 University of California at Santa Cruz Conference on Advanced Research in VLSI, 1991, pp. 229-244.

SCHN88 Schneider, B.O. and U. Claussen, "PROOF: An Architecture for Rendering in Object-Space," Advances in Computer Graphics Hardware III, Eurographics Seminars, 1988, pp. 121-140.

WEIN81 Weinberg, R., "Parallel Processing Image Synthesis and Anti-Aliasing," SIGGRAPH '81, Vol. 15, No. 3, pp. 55-61. 